\title{
Current Status of the Management of Hereditary Breast and Ovarian Cancer in Asia: First Report by the Asian BRCA Consortium
}

\author{
Seigo Nakamura ${ }^{1}$, Ava Kwong ${ }^{2 \star}$, Sung-Won Kim ${ }^{3}$, Philip Iau ${ }^{4}$, \\ Pimpicha Patmasiriwat ${ }^{5}$, Rodney Dofitas ${ }^{6}$, Teguh Aryandono ${ }^{7}$, Zhen $\mathrm{Hu}^{8}$, \\ Chiun-Sheng Huang ${ }^{9}$, Ophira Ginsburg ${ }^{10}$, Muhammad Usman Rashid ${ }^{11}$, \\ Rajiv Sarin ${ }^{12}$, Soo-Hwang Teo ${ }^{13}$ \\ ${ }^{1}$ Division of Breast Surgical Oncology, Department of Surgery, Showa University School of \\ Medicine, Japan \\ ${ }^{2}$ Department of Surgery, The University of Hong Kong, Hong Kong \\ ${ }^{3}$ Department of Surgery, Seoul National University Bundang Hospital, Korea \\ ${ }^{4}$ Department of Surgery, National University of Singapore, Singapore \\ ${ }^{5}$ Department of Clinical Microscopy, Mahidol University, Thailand \\ ${ }^{6}$ Department of Surgery, University of the Philippines Manila - Philippine General Hospital, \\ Philippines \\ ${ }^{7}$ Department of Surgery, Gadjah Mada University, Indonesia \\ ${ }^{8}$ Department of Breast Surgery, Fudan University, China \\ ${ }^{9}$ Department of Surgery, National Taiwan University Hospital, Taiwan \\ ${ }^{10}$ Department of Medicine, University of Toronto, Canada \\ ${ }^{11}$ Department of Basic Sciences Research, Shaukat Khanum Memorial Cancer Hospital and \\ Research Centre, Pakistan \\ ${ }^{12}$ Department of Radiation Oncology and Genetics, Tata Memorial Center, India \\ ${ }^{13}$ Department of Surgery, University Malaya Cancer Research Institute, Malaysia
}

Background/Purpose: $B R C A 1 / 2$ mutation carriers possess an elevated lifetime risk for hereditary breast and ovarian cancer (HBOC), yet the availability of risk assessment and genetic testing for HBOC in Asians is far limited, thus limiting the chances for appropriate surveillance, clinical strategies and cancer management.

Methods: Current status of HBOC management in 14 Asian countries was reviewed. Data of genetic counseling and genetic testing uptake rates were collected, and were analyzed how economic factors, healthcare system, legal frameworks and cultural issues affect the genetic service availability in Asia.

Results: It was estimated that in 2012, of the 560,000 breast cancer patients in 14 Asian countries, only around 4,000 individuals were benefited from genetic counseling and 3,200 were tested, in which over $40 \%$ of the cases were from Korea. Economic factors 
such as genetic service costs, and the lack of adoption into national healthcare systems (with the exception of Korea) are major barriers to genetic risk assessment in Asian countries. In addition, regional accredited laboratories, training programmes, and trained healthcare professionals are readily available only in half of the studied countries.

Conclusion: These limitations in healthcare system, combined with the lack of legal frameworks against genetic discrimination and lack of awareness among general public, remain the main challenges to HBOC management in Asia. The Asian BRCA (ABRCA) Consortium reports the current limitations in genetic counseling and testing for familial breast cancer in Asia, and urges the governments, healthcare sectors and researchers to address the disparities in HBOC management. 